\title{
Patología del uraco: revisión de conjunto y presentación de tres casos
}

\author{
M.J. Donate Moreno, J.M. Giménez Bachs, A.S. Salinas Sánchez, J.G. Lorenzo Romero, \\ M. Segura Martín, I. Hernández Millán, J.Mª Pastor Guzmán, R. Ruiz Mondéjar, \\ J. Virseda Rodríguez.
}

Servicio de Urología. Complejo Hospitalario y Universitario de Albacete. Albacete..

Actas Urol Esp 2005; 29 (3): 332-336

\section{RESUMEN}

PATOLOGÍA DEL URACO: REVISIÓN DE CONJUNTO Y PRESENTACIÓN DE TRES CASOS

Con la presentación de estos dos casos (quiste y adenocarcinoma de uraco) realizamos una revisión de conjunto de la patología uracal.

El quiste de uraco suele tener un curso asintomático, descubriéndose de manera casual al realizar otras pruebas diagnósticas o cuando surgen complicaciones del mismo.

$\mathrm{El}$ adenocarcinoma uracal es una entidad rara, se presenta habitualmente con hematuria debiendo seguir el mismo protocolo diagnóstico que para los tumores vesicales (cistoscopia y RTU-vesical). Ha de plantearse el diagnóstico diferencial con el adenocarcinoma de cúpula vesical. La cistectomía parcial es el tratamiento de elección. La quimioterapia y radioterapia ofrecen pobres resultados.

Palabras clave: Patología uracal. Quiste uraco. Adenocarcinoma uraco. Uraco.

\section{ABSTRACT}

URACHAL PATHOLOGY: AN OVERVIEW REVISION AND PRESENTATION OF THREE CLINICAL CASES

With the use of these two clinical cases (cyst and urachal adenocarcinoma) we did an overview of the urachal pathology.

The urachus cyst is usually asyntomathic, it's detected randomly when we do other diagnostic tests or when we have any complications.

The urachal adenocarcinoma is a rare pathology, it usually exhibit hematuria and we need to follow the same diagnostic tools as we use in vesical tumors (cystoscopy and transurethral vesical resection). Adenocarcinoma of the dome of the bladder is the main differential diagnosis. Partial cystectomy is the first choice treatment. Quimiotheraphy and radiotheraphy offer poor results.

Keywords: Urachal pathology. Urachus cyst. Urachal adenocarcinoma. Urachus.

$\mathrm{E}^{1}$ uraco es un cordón fibroso obliterado, resto de la alantoides, que transcurre desde la cúpula vesical hasta el ombligo, situado en el espacio de Retzius entre la fascia transversalis y el peritoneo, limitado lateralmente por las dos arterias umbilicales involucionadas.

Como consecuencia del fallo en el proceso de obliteración de la luz uracal se producen los diferentes tipos de anomalías del uraco: uraco per- meable congénito, fístula uracal, divertículo uraco-vesical, quiste de uraco y sinus alternante. Presentamos un caso de quiste de uraco asintomático y otro infectado.

Los tumores malignos del uraco son muy poco frecuentes y debe plantearse el diagnóstico diferencial con el adenocarcinoma de cúpula vesical. Aportamos un caso de adenocarcinoma de uraco $\mathrm{y}$ analizamos cuestiones sobre su diagnóstico y tratamiento. 


\section{CASOS CLÍNICOS}

Caso $n^{-} 1$ (Quiste de uraco)

Mujer de 32 años, sin ninguna patología médica ni intervenciones quirúrgicas previas que se realiza una ecografia abdominal de control durante su embarazo. En esta se aprecia una masa quística supravesical, en el trayecto del uraco, en íntimo contacto con la cúpula vesical, homogénea y con contenido en su interior. La paciente no había presentado ningún síntoma relacionado con este hallazgo ecográfico casual. Se decide dejar transcurrir su embarazo y tras el parto se realiza RMN abdominal para mejor valoración del caso (Fig. 1). Con el diagnóstico de presunción de quiste de uraco se propone intervención quirúrgica, realizando laparotomía media infraumbilical, procediéndose a la exéresis de una pieza de $11 \times 5,5$ $\mathrm{cm}$., situada entre la fascia transversalis y el peritoneo, conectada superiormente con el ombligo e inferiormente con la vejiga a través de un tejido fibromuscular. Al corte, presentaba características quísticas, con un contenido mucinoso amarillento en su interior (Fig. 2).

\section{Caso no 2 (Quiste de uraco infectado)}

Varón de 17 años de edad, sin ningún antecedente de interés. Acude a Urgencias por un cuadro de fiebre, dolor hipogástrico y un síndrome

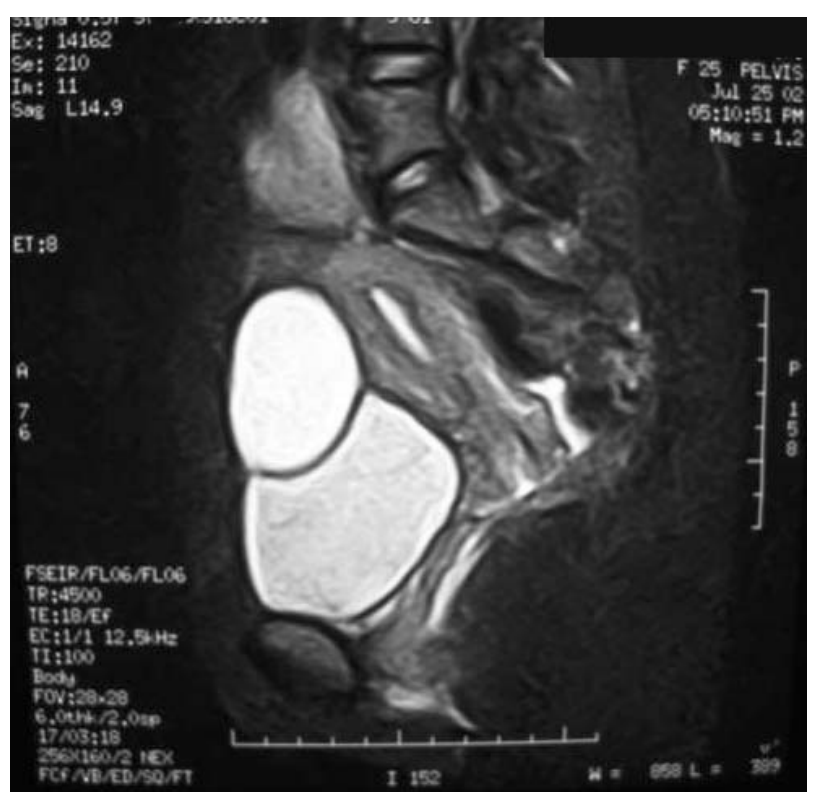

FIGURA 1. Masa quistica en la pared anterior del abdomen, en contacto con la vejiga y que la desplaza inferiormente, sugestiva de quiste de uraco.

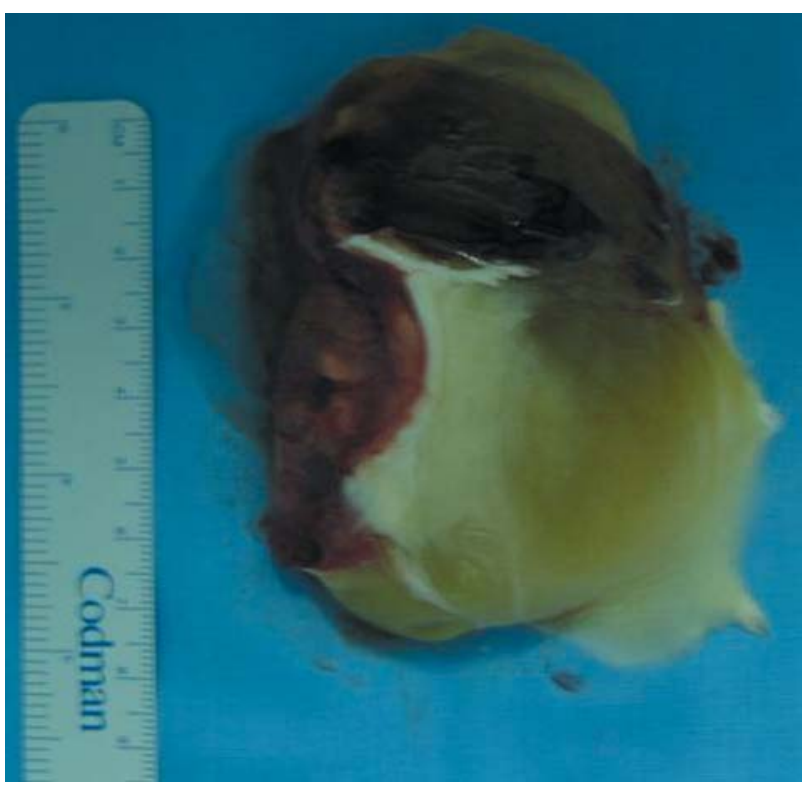

FIGURA 2. Pieza quirúgica.

miccional de características irritativas. En la analítica sanguínea destaca leucocitosis con desviación a la izquierda y en la urinaria piuria y bacteriuria. Se realiza ecografia abdominal apreciándose una masa hipogástrica quística con contenido en su interior que parece conectar con la cúpula vesical. Para mejor valoración del caso se realiza TAC abdomino-pélvico donde se objetiva una masa supravesical, en el trayecto del uraco,homogénea y con contenido en su interior. Con el diagnóstico de quiste uracal infectado se programa para intervención quirúrgica realizando laparotomía media infraumbilical procediendo a excisión completa de los restos uracales.

\section{Caso no 3 (Adenocarcinoma de uraco)}

Varón de 73 años de edad, con antecedentes de cardiopatía isquémica intervenida con revascularización de 3 vasos e hipertenso en tratamiento médico. Consulta en el servicio de urología por hematuria. En UIV se aprecia una vejiga trabeculada con alguna imagen diverticular y con un defecto de replección central por una tumoración intravesical. Ecografia abdominal (Fig. 3): formación ecograficamente quística en hipogastrio, en el trayecto teórico del uraco, compleja y heterogénea con áreas de mayor ecogenicidad que parecen depender de la pared, demostrándose una pequeña comunicación con la vejiga a nivel del margen izquierdo de la cúpula vesical. 


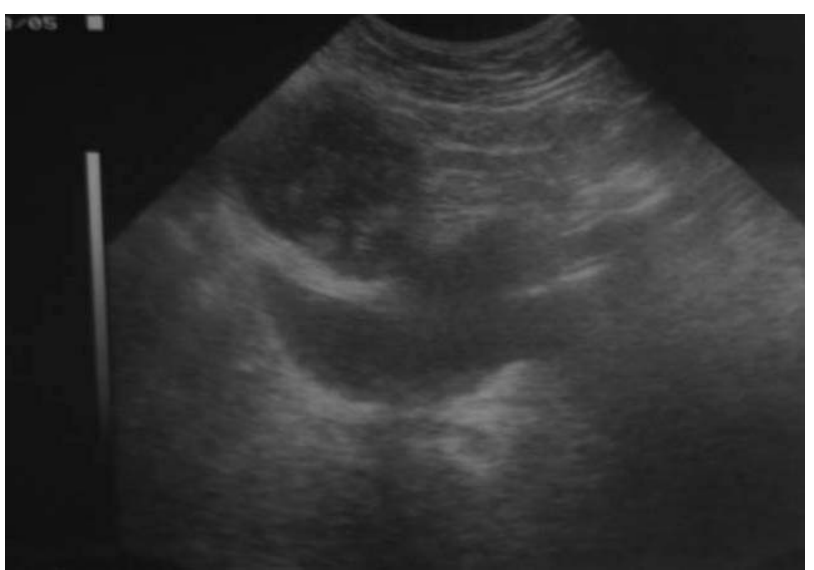

FIGURA 3. Ecografia abdominal.

Se practica exploración vesical bajo anestesia y RTU vesical para toma de biopsias donde se observa una lesión sobreelevada en cúpula de tipo papilar con necrosis y contenido mucoso en su interior, sugestiva de lesión quística o diverticular. El resultado anatomopatológico de la biopsia demuestra abundante material mucoide con escaso componente epitelial. Histológicamente dicho componente muestra papilas revestidas por epitelio columnar mucosecretante con pseudoestratificación y atipia. Se completa el estudio con un TAC abdómino-pélvico: en hipogastrio pelvis menor se objetiva una formación quística bien delimitada de aproximadamente $6 \mathrm{~cm}$ de diámetro, con una porción más inferior de aspecto sólido que realza con contraste intravenoso. Dicha lesión parece contactar con la superficie interna de los músculos rectos anteriores del abdomen y se extiende caudalmente hacia una localización supravesicalextravesical con impronta en cúpula vesical de semiología más sólida.

Ante esta situación se plantea intervención quirúrgica, realizando laparotomía media infraumbilical-extraperitoneal y practicando cistectomía parcial (Fig. 4). El resultado anatomopatológico de la pieza de cistectomía es informado como adenocarcinoma que crece con patrones enteroide y mucinoso de origen uracal.

\section{DISCUSIÓN}

El uraco se desarrolla en la vida fetal temprana como una conexión tubular desde el esbozo vesical hasta la alantoides en el ombligo. Cuando la vejiga desciende a la pelvis, entre los 4 y 5

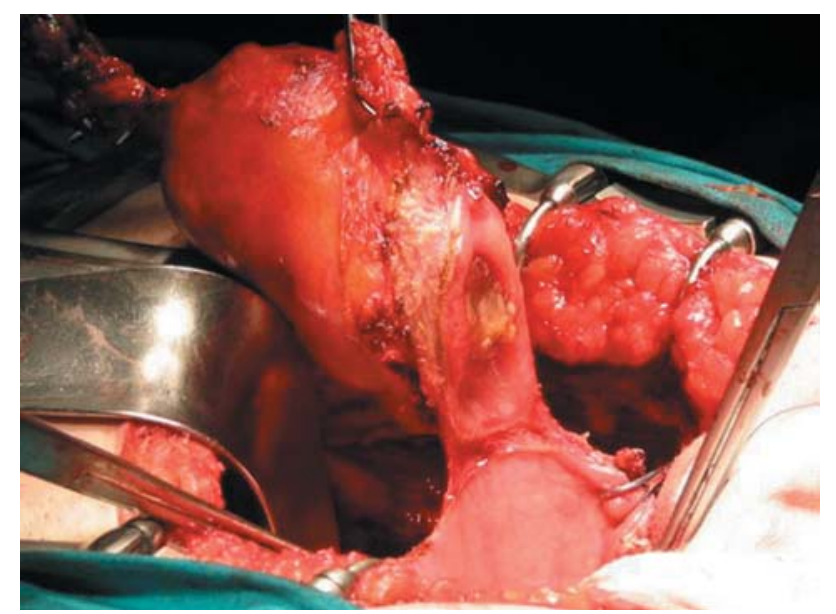

FIGURA 4. Imagen que muestra un momento de la cirugia de exéresis del adenocarcinoma de uraco.

meses de gestación, el uraco involuciona y se oblitera formando un cordón fibromuscular. $\mathrm{Su}$ longitud en el adulto oscila entre los 3 y $10 \mathrm{~cm}$ de largo y su diámetro entre 8 y $10 \mathrm{~mm}$, y está situado en el tejido conectivo del espacio extraperitoneal de Retzius, con la fascia transversalis como límite anterior, el peritoneo parietal en la cara posterior y los ligamentos umbilicales, que son a su vez remanentes de las arterias umbilicales fetales dispuestos a ambos lados ${ }^{1-3}$.

Histológicamente el uraco está constituido por tres capas diferentes: una interna de epitelio transicional, una capa intermedia de tejido conectivo y una externa de músculo liso ${ }^{1-3}$.

Se describen dos grupos de patología uracal: congénita y adquirida. El uraco permeable congénito consiste en la persistencia de la comunicación fetal entre la cúpula vesical y el ombligo, manifestándose con la salida de orina por el ombligo desde el nacimiento. Las anomalías uracales adquiridas se caracterizan por la reapertura parcial de la luz uracal: a) fístula uracal, quiste comunicado con el ombligo; b) divertículo uraco-vesical, estructura quística comunicada con la vejiga; c) quiste de uraco, no comunicado ni con la vejiga ni con el ombligo; d) fístula alternante, es un quiste de uraco que drena intermitentemente a la vejiga o al ombligo ${ }^{1,4,5}$.

Es difícil concretar las cifras exactas de incidencia y prevalencia de la afección uracal debido a que habitualmente siguen un curso asintomático. Aunque puede aparecer a cualquier edad lo habitual es que se presente durante la infancia ${ }^{3}$. 
El quiste de uraco es una alteración producida al obliterarse los extremos uracales (vesical y umbilical), persistiendo una cavidad quística intermedia con líquido seroso o mucinoso en su interior resultado de la secreción y descamación de las células epiteliales uracales $^{6}$. Su localización habitual es en el tercio inferior del uraco, sin que produzca sintomas en la mayoría de los casos. Generalmente se descubren de forma casual al realizar pruebas diagnósticas o cuando producen complicaciones (infección, litiasis, repermeabilización o degeneración neoplásica) ${ }^{7}$.

El estudio preoperatorio se puede realizar con variedad de pruebas de imagen. Gracias a la accesibilidad del uraco, localizado en la pared anterior del abdomen, lejos de la interferencia del gas intestinal, la ecografía es una técnica muy apropiada para una buena aproximación diagnóstica. La TAC es la prueba de imagen que proporciona mayor exactitud, tanto para la visualización y delimitación de la cavidad quística uracal, como para el estudio de las estructuras adyacentes, permitiendo realizar un diagnóstico diferencial con otras patologías abdomino-pélvi$\operatorname{cas}^{3,8}$. Otra prueba que también se debe incluir es una urografía intravenosa por la posibidad de anomalías asociadas del tracto urinario; así, el $43 \%$ de los pacientes con anomalías uracales presentan alteraciones genitourinarias asociadas, como reflujo vesicoureteral, riñón único, criptorquidia, síndrome de la unión pieloureteral o hidronefrosis $^{3,5}$. La cistouretrografía miccional se puede realizar también para analizar la zona de comunicación entre la vejiga y el uraco y la posible compresión de la cúpula vesical ${ }^{3}$. En adultos esta indicada la realización de cistoscopia para descartar un carcinoma uracal ${ }^{8}$.

La cirugía, con la excisión completa de los restos uracales es el tratamiento indicado ${ }^{4}$. En manos de cirujanos expertos y con pacientes cuidadosamente seleccionados se podría realizar la cirugía laparoscópica, proporcionando una menor estancia hospitalaria y una más rápida recuperación ${ }^{9,10}$.

El adenocarcinoma de uraco es una entidad tumoral poco frecuente, se describe una incidencia media de 1 caso por 5 millones de habitantes, que predomina en varones, con una relación 4/1 con respecto a las mujeres ${ }^{11}$.
En cuanto a su histogénesis, Mostoficitado12-15 establece unos criterios diferenciales entre adenocarcinomas uracales y vesicales primarios: a) el tumor se localiza en la cúpula vesical con desarrollo extravesical o intramural; b)mucosa vesical adyacente normal con ausencia de áreas de cistitis quística o glandular; c) no evidencia de tumor primario en otra localización; d) existencia de restos uracales con tumor o crecimiento del tumor hacia la vejiga con extensión al espacio de Retzius, pared anterior del abdomen o al ombligo. Otra clasificación histológica divide a los adenocarcinomas de uraco en tubulares, papilares, mucinosos o coloides y adenocarcinomas en anillo de sello. Como en nuestro caso, la mayoría de los tumores son adenocarcinomas mucinosos. Precisamente son estas características histológicas las que se relacionan con la supervivencia, siendo los tumores mucinosos los que tienen mejor pronóstico en comparación con el resto de subtipos $^{11,16}$.

Resulta complicado el diagnóstico precoz debido a la ausencia de manifestaciones clínicas en los estadios iniciales. La clínica suele derivar de la invasión del tumor de estructuras vecinas. El síntoma más frecuentemente presentado es la hematuria, seguido de disuria, dolor-sensación de peso hipogástrico o expulsión de contenido mucoide por la orina ${ }^{12,13}$. Al diagnóstico llegaremos por una vía similar a la seguida en los tumores vesicales: la cistoscopia y la resección transuretral son fundamentales. En la radiografía simple de aparato urinario podríamos encontrar frecuentemente calcificaciones. En la UIV se aprecian defectos de replección en la cúpula vesical. En la ecografía se observa una masa supravesical con ecos en su interior. TAC y RMN son fundamentales a la hora de planificar el tratamiento quirúrgico puesto que nos dan idea de las relaciones entre la tumoración y las estructuras contigüas en cuanto a su posible afectación y a la existencia de ganglios linfáticos regionales ${ }^{17}$. Ante la sospecha de adenocarcinoma uracal siempre se debe descartar la posibilidad de que sea metastático, excluyendo patología prostática (PSA,PAP y biopsia prostática), del aparato genital femenino o posible origen gastrointestinal ${ }^{13}$. Se han descrito algunas técnicas imnunohistoquímicas como criterios para realizar diagnóstico 
diferencial entre adenocarcinoma de uraco y el colónico: el adenocarcinoma uracal presenta positividad para CK7 y CK20, mientras que el de colon es CK7 negativo ${ }^{18}$.

Sheldon ${ }^{16}$ realizó una clasificación de estadiaje de los adenocarcinomas de uraco: Estadio I: el tumor se localiza en la mucosa uracal; Estadio II: el tumor invade la submucosa o la capa muscular del uraco pero se encuentra localizado en el uraco; Estadio III: el tumor se extiende fuera del uraco, IIIA a la vejiga, IIIB a la pared abdominal, IIIC al peritoneo y IIID a alguna víscera diferente; Estadio IV: tumor con metástasis a distancia.

El tratamiento indicado de los tumores uracales es la cistectomía parcial extensa, con excisión en bloque de los tejidos del uraco, desde vejiga hasta el ombligo, de la lámina posterior de la vaina de los rectos, así como la realización de una linfadenectomía pélvica bilateral ${ }^{19-21}$.

El pronóstico no es bueno, ya que la mayoría se diagnostican en estadios avanzados. Entre el 43 y el 50\% de los casos sobreviven a los 5 años tras la cirugía. Estos tumores son considerados radioresistentes y presentan una dudosa quimiosensibilidad $^{22}$.

\section{REFERENCIAS}

1. Mesrobian HG, Zacharias A, Balcom Ah, Cohen Rd. Ten years of experience with isolated urachal anomalies in child. J Urol 1997;158:1316-1318.

2. Hernández-Siverio N, Rodríguez P, Robayna G. Quistes de uraco. Estado actual del problema. Arch Esp de Urol 1990; 43(3):237-239.

3. Montserrat Orri V, Mus Malleu A, Gutiérrez Sanz-Gadea C, Sala O`Shea E, Conte Visus A, Ozonas Moragues M. Patología benigna del uraco en el adulto: a propósito de dos observaciones. Actas Urol Esp 1990;14(3):217-220.

4. Berman SM, Tolia BM, Laor E, Reid RE, Schweizerhof SP, Freed SZ. Urachal remnants in adults. Urology 1988;31 (1):17-21.

5. Macneily AE, Koleilat N, Kiruluta HG, Homsy YI.: Urachal abscesses: protean manifestations, their recognition and management. Urology 1992;40(6):530-535.

6. Kilani Elmasri S, Albors Valls L, Monsalve Rodríguez M, Gómez Cisneros S, Parra Mountaner L, García Alonso J.: Quiste uracal infectado en adultos. Actas Urol Esp 1994;18 (4):312-314.

7. Gomez Parada J, Puyol Pallas JM.: Quiste infectado de uraco: a propósito de un nuevo caso. Arch Esp Urol 2001;54 (7):722-725.
8. Iuchtman M, Rahav S, Zer M, Mogilner J, Siplovich L. Management of urachal anomalies in children and adults. Urology 1993;42:426-430.

9. Jeffrey A. Cadeddu, Karen E. Boyle, Michael D. Fabrizio, Peter G. Schulam, Louis R. Kavoussi. Laparoscopic management of urachal cysts in adulthood. J Urol 2000;164: 1526-1528.

10. Stone N, Garden R, Weber H. Laparoscopic excision of urachal cyst. Urology 1995;45(1):161-164.

11. Ojea Calvo A, Núñez López A, Domínguez Freire F, Alonso Rodrigo A, Rodríguez Iglesias B, Benavente Delgado J, Barros Rodríguez JM, González Piñeiro A, Otero García M, López Bellido D.: Adenocarcinoma mucinoso de uraco. Actas Urol Esp. 2003;27(2):142-146.

12. Salinas Sánchez AS, Alcalá-Santaella Casanova C, Martínez Martín M, Pastor Guzmán JM, Cañamares Pabolaza L, Virseda Rodríguez J. Adenocarcinomas de uraco. Arch Esp Urol. 1991;44(1):31-36.

13. Barros Rodríguez JM, Fernández Martín R, Guate Ortiz Jl, Ojea Calvo A, Machuca Santa-Cruz J, Mata Varela J, Jamardo González D, Figuereido Godoy L, Nogueira March Jl.: Adenocarcinoma mucinoso de uraco. Actas Urol Esp. 1989;13(5):399-401.

14. González Enguita C, Martínez Bengoechea J, Gil Sanz Mj, Rodríguez Vela L, Cavero Rebollo O, Cay Diarte A, Roncales Badal A, Liédana Torres JM, Rioja Sanz LA.: Adenocarcinoma vesical de origen uracal. Un raro tumor. Actas Urol Esp. 1991;15:278-282.

15. Palmero Marti Jl, Queipo Zaragoza JA, Bonillo García MA, Budia Alba A, Vera Sempere FJ, Jiménez Cruz JF: Adenocarcinoma mucinoso de vejiga. Actas Urol Esp 2003; 27(4):274-280.

16. Sheldon CA, Clayman RV, González R et al. Malignant urachal lesions. J Urol 1984;131:1-8.

17. Mengiardi B, Wiesner W, Stoffel F, Terracciano L, Freitag P. Adenocarcinoma of the urachus.: Radiology 2002;222(3): 744-747.

18. Álvarez Álvarez C, Sánchez Merino JM, Busto Castañón L, Pombo Felipe F, Arnal Monreal F. Adenocarcinoma mucinoso de uraco sincrónico con carcinoma colorrectal. Valor de la inmunohistoquímica en el diagnóstico diferencial. Actas Urol Esp 1998;22:515-518.

19. Henly DR, Farrow GM, Zincke H. Urachal cancer: role of conservative surgery. Urology 1993;42(6):635-639.

20. Santucci RA, True LD, Lange PH. Is parcial cystectomy the treatment of choice for mucinous adenocarcinoma of the urachus?. Urology 1997;49(4):536-540.

21. Herr HW. Urachal carcinoma: the case for extended partial cystectomy. J Urol 1994;151(2):365-366.

22. Siefker-Radtke AO, Gee J, Shen Y, Wen S, Daliani D, Millikan RE, Pisters LI. Multimodality management of urachal carcinoma: the M.D Anderson Cancer Center experience. J Urol 2003;169:1295-1298.

Dra. M.J. Donate Moreno

C/ Ávila $\mathrm{n}^{\circ} \mathrm{17}, 4^{\circ} \mathrm{G}$

2002 Albacete

(Trabajo recibido el 3 noviembre de 2004) 